\title{
Development of High Performance Filter for Indoor Positioning System
}

\author{
Naoya Muramatsu ${ }^{\text {a, }}$, CW. Ooi ${ }^{a}$, Takashi Miyazaki ${ }^{b}$ \\ ${ }^{a}$ University of Tsukuba, 1-1-1 Tennodai, Tsukuba, Ibaraki, 305-8577, Japan \\ ${ }^{b}$ National Institute of Technology, Nagano College, 716 Tokuma, 381-8550 Nagano, Japan \\ *Corresponding Author: sh.mn.nat@gmail.com
}

\begin{abstract}
In indoor environment where GPS cannot be used, various methods for measuring the position information of objects have been proposed. Among them, the low introduction cost and effectiveness of the method using reception strength of the Wi-Fi or the wireless module is drawing attention. However, the method using Received Signal Strength Indicator(RSSI) or Link Quality Indicator (LQI) is greatly affected by radio noise. We propose a filter which greatly suppresses the influence of radio noise in indoor position information system using LQI value of radio waves. We also clarified that the influence of wireless noise is greatly reduced by implementing and applying the system when the physical position of the beacon is stationary.
\end{abstract}

Keywords: IoT, Radio noise, Noise filter, Indoor positioning system.

\section{Introduction}

The location-based service (LBS) provided in the ubiquitous environment requires the user's exact location, and as a result, the location information technology is one of the most important elements in the ubiquitous network ${ }^{(1)}$. The most representative technology is the Global Positioning System (GPS).

It is widely used in practical outdoor LBS system. However, when the visibility of the line of sight to the satellite is lost, GPS signals cannot be received fully, thus GPS cannot be used indoors as precise.

Many indoor positioning technologies have recently been developed to enable indoor LBS. Active Badge ${ }^{(2)}$ and Cricket ${ }^{(3)}$ including positioning utilizing the difference between the propagation time of ultrasonic wave and RF signal, Active Badge which detects the infrared signal and performs positioning. Positioning using the intensity of the received UDP signal is one of the most representative indoor positioning systems. These systems are very accurate, but they also have their own drawbacks. That is, they require a special device dedicated to positioning which adds to the cost. Many indoor positioning systems that do not require special equipment have also been developed. Most of them use RF based WLAN (Wireless LAN) positioning technology. Today WLAN can be used in various places such as university campus, airport, hotel, and even home. The indoor positioning system introduced in this paper is also a kind of RF based WLAN positioning system. An RF-based WLAN positioning system determines the position of a user by referencing to the LQI of the signals from the various access points (APs). The most common method used to determine the position of a user is the fingerprinting method ${ }^{(4-6)}$. In the implementation of the fingerprinting method, any classification and decision making methods as shown in $(7,8)$ can be applied. The development of positioning systems based on fingerprinting consists of two phases. First, in the offline phase, positional fingerprints are collected by performing site survey of LQI from multiple APs. The vector of LQI values at a point is called the position fingerprint of that point. In the second phase, the online phase, the LQI received by the user at the present time are collected and the user's position is determined according to these fingerprints.

Fingerprinting is known to be fairly accurate. However, it has some serious drawbacks. That is, the offline phase is very time-consuming. Another common method is the method based on the RF propagation loss model ${ }^{(9,10)}$. The RF propagation loss model is a simple mathematical expression expressing the relationship between the LQI and the distance between the sender and the receiver. However, LQI is influenced by many 
parameters, establishing an appropriate RF propagation loss model is very difficult. As a result, the positioning method based on the RF propagation loss model has lower accuracy than the fingerprint positioning method. We propose WLAN positioning method based on RF propagation loss model to avoid time consuming offline phase process.

The proposed method uses a mobile terminal in a RF based WLAN positioning system that measures the strength of a signal received from at least three different fixed position locating stations. Next, the mobile terminal estimates the distance from the stations by applying the RF propagation loss model to these signal intensities. Then the mobile terminal can estimate its position by applying trilateration to station distances and coordinates. Distribution of indoor position estimated by trilateration is usually quite large due to the noise of the RF signal that caused by movement of objects or other wireless modules using the same channel pass through the detection area. In order to obtain a more accurate position from a noisy distance measurement, the terminal repeats the estimation process many times and determines that position as the average of the estimation.

Since the radio wave condition in the surrounding space changes due to the surrounding objects and the movement of humans, the estimated position may change unintentionally even when the object is stationary. In this paper, we propose a filter which greatly suppresses the influence of noise of RF signal by utilizing the change of radio wave intensity during triangulation. We also evaluated its effectiveness by building an actual indoor positioning system.

\section{System overview}

Our system consists of several compact power-saving wireless modules. The devices are Mono Wireless Inc. MONOSTIC-C $1{ }^{\mathrm{i}}$ and TWE-L-2525A ${ }^{\mathrm{ii}}$. The wireless network configuration is shown in Fig. 3. There are three functions, "Parent", "Router", and "Beacon", respectively in the network. The network has three layers namely Parent, Router, and Beacon (or End device). The Parent and the Router can communicate directly via other Routers. In addition, the Parent and the Beacon can also communicate

\footnotetext{
i https://mono-wireless.com/jp/products/MoNoStick/index.html

https://mono-wireless.com/jp/products/TWE-Lite-2525A/index.ht $\mathrm{ml}$
}

via Routers. Therefore, we can measure the LQI values of all communications performed in the whole network via Parent.

Parent is connected to the host computer in order to record, process and display information on acquired wireless network.

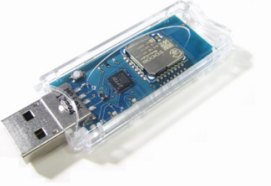

(a) MONOSTIC-C1

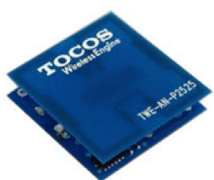

(b) TWE-L-2525A
Fig. 1. Compact power saving wireless modules.

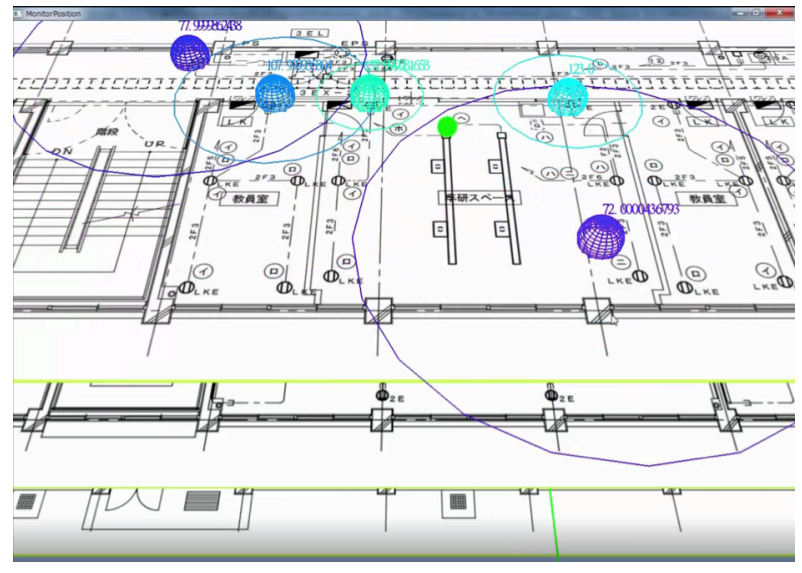

Fig. 2. Execution screen of the installed position information system: a mesh sphere indicates the position of each Router, and its color corresponds to the LQI value. The yellowish green sphere in the center shows estimated position information of Beacon.

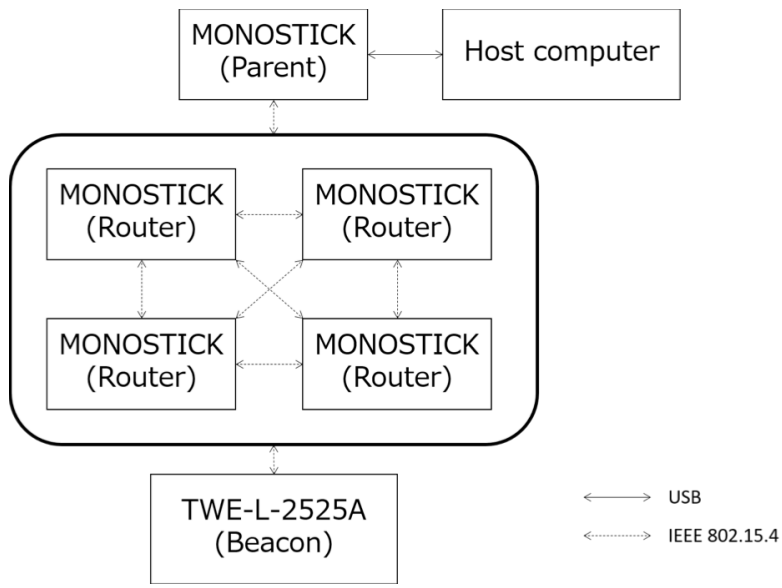

Fig. 3. The wireless network configuration. 


\section{Simply Filter based on Triangulation}

\subsection{Triangulation}

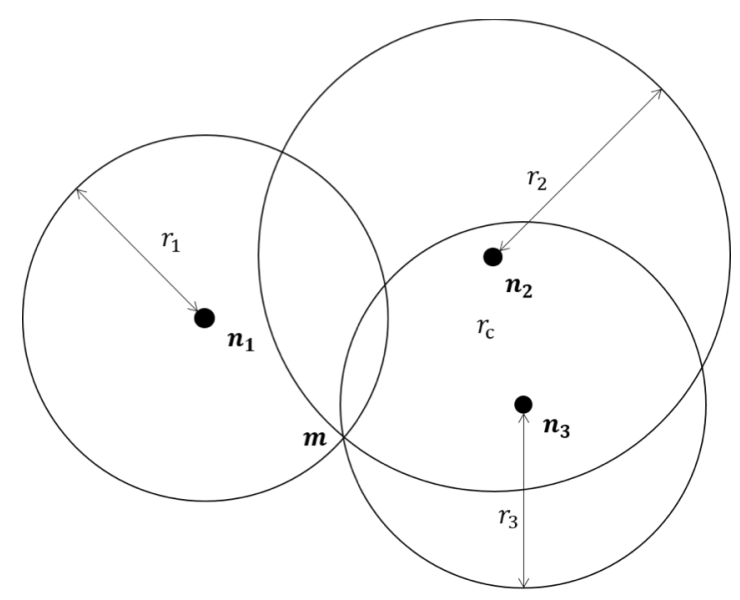

Fig. 4. A diagram to illustrate trilateration.

As shown in Fig. 4, when $N$ range $r_{1}, r_{2}, \ldots, r_{N}$ from $N$ base stations $\boldsymbol{n}_{\mathbf{1}}=\left(X_{1} Y_{1} Z_{1}\right)^{\mathrm{T}}, \ldots, \boldsymbol{n}_{\boldsymbol{N}}=\left(X_{N} Y_{N} Z_{N}\right)^{\mathrm{T}}$ to mobile terminal $\boldsymbol{m}=\left(\begin{array}{lll}x y & y\end{array}\right)^{\mathrm{T}}$ is measured, coordinates of $m$ can be estimated using trilateration. By squaring it we obtain the following equation for $r_{i}^{2}$ :

$$
\begin{aligned}
\left(x-X_{i}\right)^{2}+\left(y-Y_{i}\right)^{2}+\left(z-Z_{i}\right)^{2} & =r_{i}^{2} \\
(\text { for } i & =1,2, \ldots, N)
\end{aligned}
$$

By subtracting $r_{i}^{2}$ from $r_{i}^{2}(i=2, \ldots, \mathrm{N})$, we have $A \boldsymbol{x}=\boldsymbol{b}$, where

$$
\begin{gathered}
A=2\left[\begin{array}{ccc}
\left(X_{2}-X_{1}\right) & \left(Y_{2}-Y_{1}\right) & \left(Z_{2}-Z_{1}\right) \\
\vdots & \vdots & \vdots \\
\left(X_{N}-X_{1}\right) & \left(Y_{N}-Y_{1}\right) & \left(Z_{N}-Z_{1}\right)
\end{array}\right], \boldsymbol{x}=\left[\begin{array}{c}
x \\
y \\
Z
\end{array}\right] \\
\boldsymbol{b}=\left[\begin{array}{c}
\left(X_{2}^{2}-X_{1}^{2}\right)+\left(Y_{2}^{2}-Y_{1}^{2}\right)+\left(Z_{2}^{2}-Z_{1}^{2}\right)-\left(r_{2}^{2}-r_{1}^{2}\right) \\
\vdots \\
\left(X_{N}^{2}-X_{1}^{2}\right)+\left(Y_{N}^{2}-Y_{1}^{2}\right)+\left(Z_{N}^{2}-Z_{1}^{2}\right)-\left(r_{N}^{2}-r_{1}^{2}\right)
\end{array}\right]
\end{gathered}
$$

Due to the fact that coordinates are 3 dimensional, we need to have at least 4 base stations. When there are two base stations that receive signals from the beacon, we can estimate the location of $m, \widehat{x}$, with the received position coordinates by using the MMSE (Minimum Mean Square Error) method:

$$
\widehat{\boldsymbol{x}}=\left(A^{\mathrm{T}} A\right)^{-1} A^{\mathrm{T}} \boldsymbol{b}
$$

\subsection{Overview of Simply Filter}

The positioning system based on the LQI values is sensitive to the influence of radio noises. If the target (user's real position) stand still, the position estimated from the result of triangulation calculation using the LQIs between receiver and base stations is not stable because the LQI values between some base stations and the receiver randomly fluctuates.

In order to reduce the influence of the radio noises, we propose a simple and effective method exploiting the simple features of radio noise. The filter is designed based on the hypothesis that "the noise randomly occurs in the communication between a Router and the Beacon and the probability of occurrence of noise at the same time for multiple communications is low". We also use the median filter.

Assuming $n$ Routers are able to receive the Beacon radio waves, LQI values of $i$-th Router for the last three transmissions are $\left(a_{1}^{i}, a_{2}^{i}, a_{3}^{i}\right)$ with $a_{i 1}$ is the latest LQI value with the $i$-th Router. The LQI values of all Routers and a Beacon can be measured at regular intervals. In our method, $a_{\text {sum }}$ is first calculated by the following equation.

$$
\begin{gathered}
a_{\text {sum }}=\sum_{i=1}^{n}\left(\left[\begin{array}{lll}
a_{1}^{i} & a_{2}^{i} & a_{3}^{i}
\end{array}\right]\left[\begin{array}{c}
-1 \\
2 \\
-1
\end{array}\right]\right) \\
=\operatorname{sum}\left(\left[\begin{array}{ccc}
a_{1}^{1} & a_{2}^{1} & a_{3}^{1} \\
a_{1}^{2} & a_{2}^{2} & a_{3}^{2} \\
\vdots & \vdots & \vdots \\
a_{1}^{n} & a_{2}^{n} & a_{3}^{n}
\end{array}\right]\left[\begin{array}{c}
-1 \\
2 \\
-1
\end{array}\right]\right)
\end{gathered}
$$

The sum function represents the sum of each elements of the matrix.

First, we defined the $i$-th LQI value $A_{t}^{i}$ at time $t=\tau$ for use in triangulation by the following equation:

$$
A_{\tau}^{i}=\left\{\begin{aligned}
\operatorname{median}( & \left(a_{\tau}^{i}, a_{\tau-1}^{i}, a_{\tau-2}^{i}\right) \\
& \text { if } a_{\text {sum }}>t h \\
A_{\tau-1}^{i} & \text { if } a_{\text {sum }} \leq t h
\end{aligned}\right.
$$

The median function represents the median value of the matrix elements and the threshold value $t h$ is defined as $t h=k \cdot n(k \in \mathbb{R})$. In this method, the coordinates of position changes greatly and is updated unintentionally when LQI value changes so it is impossible to observe the change of fine position information.

Therefore, we obtained $A_{t}^{i}$ for each LQI values after filtering at time $t$ by the following formula.

$$
\begin{gathered}
A_{t=\tau}^{i}=a_{\tau}^{i} \varsigma_{1}(x)+a_{\text {median }}^{i}\left(1-\varsigma_{1}(x)\right) \\
x=a_{\text {sum }}-k n
\end{gathered}
$$

Here, $\varsigma_{1}(x)$ represents a standard sigmoid function, and $\varsigma_{1}(x)=\frac{1}{1+e^{-x}}$. Also, $a_{\text {median }}^{i}$ represents the median of 
$\left(a_{\tau}^{i}, a_{\tau-1}^{i}, a_{\tau-2}^{i}\right)$, and $a_{\text {median }}^{i}=\operatorname{median}\left(a_{\tau}^{i}, a_{\tau-1}^{i}, a_{\tau-2}^{i}\right)$. By using a nonlinear function such as the standard sigmoid function, the unwanted or inaccurate changes due to the threshold can be smoothened.

\section{Experiments and Results}

We applied the median filter and the filters of equations (2), (3) to the LQI values of each communication between the actual Routers and Beacon. The effectiveness of our method was clarified from experimental results. As the experimental environment, experiments were conducted in the classroom in $3^{\text {rd }}$ floor of the National Institute of Technology, Nagano College, electrical and electronic engineering building.

\subsection{Relationship between distance and LQI value}

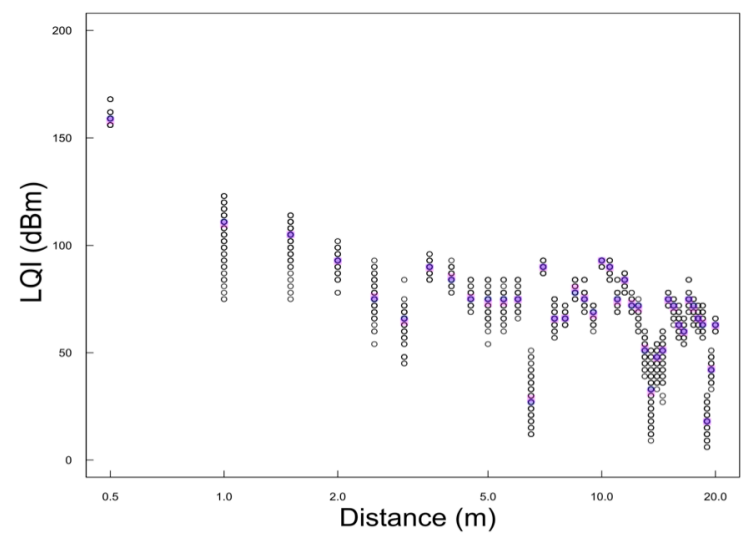

Fig. 5. Change in LQI value with distance.

In order to investigate the correspondence between the LQI value and the distance, the change in the LQI value was measured. Measurement was carried out 50 times for every $50 \mathrm{~cm}$. The measurement results are shown in Fig. 5 . The LQI value is represented by 0 to 255 , and its value is proportional to the electric field strength $y(\mathrm{dBm})$. When the LQI value is $x$, the relationship with electric field intensity $y$ is represented by $y=(7 x-1970) / 20^{i i i}$.

As shown in Fig. 5, there is a relationship between the LQI value and the device distance from 0 to $2 \mathrm{~m}$, and the LQI value decreases as the distance between the devices increases. On the other hand, when the distance is $2 \mathrm{~m}$ or more, the LQI values greatly fluctuates and become unstable. Therefore, in this system, in order to lower the implementation cost, we assume that the LQI value is

\footnotetext{
iii https://mono-wireless.com/jp/tech/Programming/Tips_LQI.html
}

directly proportional to the distance between devices.

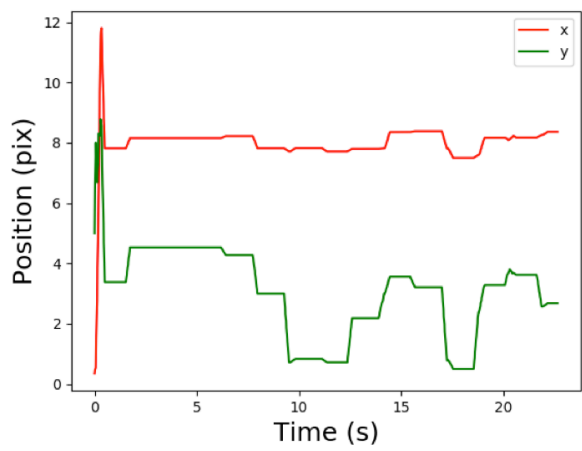

(a) Without filter

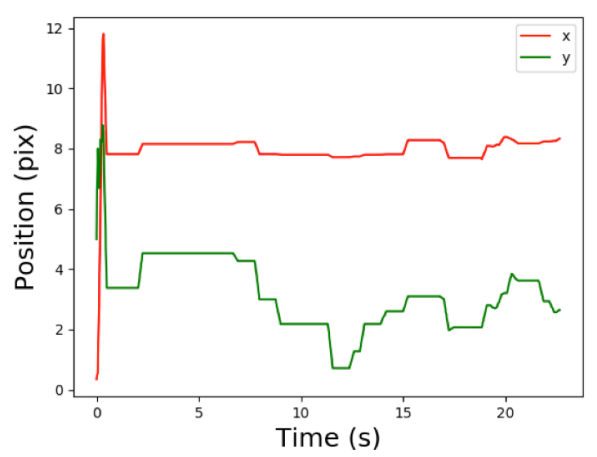

(b) Median filter

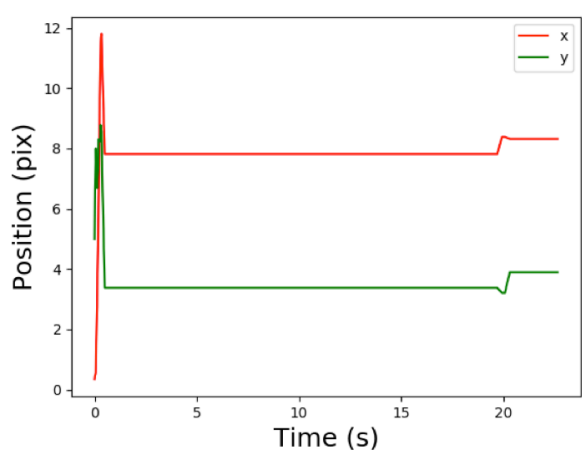

(c) The filter by threshold

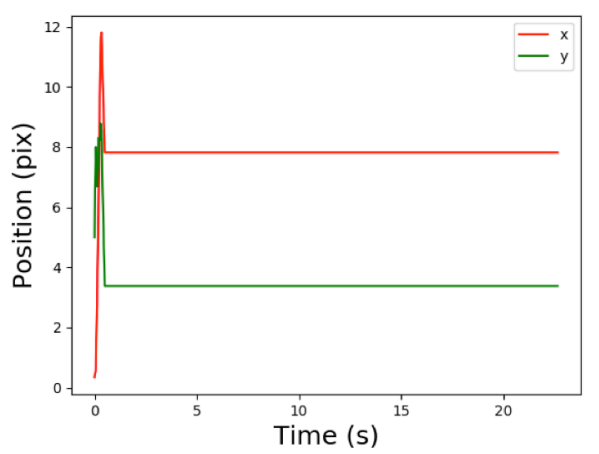

(d) The filter by standard sigmoid function

Fig 6. Change in estimated position when by each filter 


\subsection{Estimated position by filter}

We applied the median filter and the filters of equations (2), (3) to the LQI values of each communication between the actual Routers and Beacon. The effectiveness of our method was evaluated from experimental results.

Fig. 6 shows the change in the estimated position when each filter is applied. Fig. 6(a) shows the temporal change of the estimated position coordinates where filter processing is not performed. The estimated position coordinates fluctuate largely with respect to time.

Fig. 6(b), (c), and (d) use the filters introduced in Section 4.2. In Fig. 6(b), a median filter is used, which uses the median value of last three LQI values between each Routers and Beacon. It can be seen that there is almost no change compared with the result without filtering (Fig. 6(a)).

Fig. 6(c) shows the result of using the filter according to the equation (2). It can be seen that the fluctuation is largely suppressed as compared with Fig. 6(a) and (b). From this result we can see the accuracy of our method. However, the estimated position coordinates rapidly change around 20 seconds. This is because the filter based on the threshold is updating the values more frequently around the threshold.

Fig. 6(d) shows the result when using the filter according to equation (3). Further variation is suppressed as compared with Fig. 6(c). By eliminating the processing based on the threshold value using the sigmoid function, the changes are smoothened, thus the sudden fluctuations can be suppressed and it functions as a filter to radio wave noise.

\section{Conclusions}

In this paper, we propose a filter which greatly suppresses the influence of radio noise in the indoor position information system using the LQI value of radio waves. We also clarified that the influence of radio noise is greatly reduced by implementing the system and applying this method when the physical position of Beacon is stationary.

This method focuses on "the noise that randomly occurs in the communication between a Router and the Beacon" and hypothesizes that the probability of occurrence of noise at the same time for multiple communications is low. This makes it possible to create a powerful filter yet with minimal processing.

\section{References}

(1) Dae-Young Choi : "Personalized Local Internet in the Location-based Mobile Web Search", Decis. Support Syst., Vol. 43, No. 1, pp. 31-45, 2007

(2) R. Want, A. Hopper, V. Falcão and J. Gibbons : "The Active Badge Location System", ACM Trans. Inf. Syst., Vol. 10, No. 1, pp. 91-102, 1992

(3) N. Priyantha, A. Chakraborty and H. Balakrishnan : "The Cricket Location-support System", Proceedings of the 6th Annual International Conference on Mobile Computing and Networking, MobiCom '00, pp. 32-43, 2000

(4) P. Bahl and V.N. Padmanabhan : "RADAR: an in-building RF-based user location and tracking system”, INFOCOM 2000, Vol. 1, No. 1, pp. 775-784, 2000

(5) T. Lin and P. Lin : "Performance comparison of indoor positioning techniques based on location fingerprinting in wireless networks", Wireless Networks, Communications and Mobile Computing, Vol. 1, No. 1, pp. 1569-1574, 2005

(6) D. Madigan, E. Einahrawy, R.P. Martin, W. Ju, P. Krishnan and A.S. Krishnakumar : "Performance comparison of indoor positioning techniques based on location fingerprinting in wireless networks", INFOCOM 2005, Vol. 1, No. 1, pp. 1217-1227, 2005

(7) C. Antunes and L. Dias : "Managing uncertainty in decision support models foreword to the special issue", Decis. Support Syst., Vol. 43, No. 4, pp. 1451- 1453, 2007

(8) D. Power and R. Sharda : "Model-driven Decision Support Systems: Concepts and Research Directions", Decis. Support Syst., Vol. 43, No. 3, pp. 1044- 1061, 2007

(9) T. Gigl, G. Janssen, V. Dizdarevic, K. Witrisal and Z. Irahhauten : "Analysis of a UWB Indoor Positioning System Based on Received Signal Strength", Positioning, Navigation and Communication, Vol. 1, No. 1, pp. 97-101, 2007

(10) F. Lassabe, P. Canalda, P. Chatonnay and F. Spies : “A Friis-based calibrated model for WiFi terminals positioning", Proceedings of the Sixth IEEE International Symposium on World of Wireless Mobile and Multimedia Networks, Vol. 1, No. 1, pp. 382-387, 2005 